\title{
Efficiency and Benefits of Wireless Network Segregation
}

\author{
Athanasios Paraskelidis \\ School of Computing \\ Parallel Systems and Networks Research \\ Group \\ University of Portsmouth \\ United Kingdom \\ athanasios.paraskelidis@port.ac.uk
}

\author{
Dr Mo Adda \\ School of Computing \\ Parallel Systems and Networks Research \\ Group \\ University of Portsmouth \\ United Kingdom \\ mo.adda@port.ac.uk
}

\begin{abstract}
This paper examines the performance of segregate networks with single and multiple channels. It compares the simulation results to the location-aware channel assignment protocol named GRID. The term segregated means that the network is divided into smaller domains and each one operates on different radios. Each node is assigned a predefine pool of radios, per segregate. The results show that the average delay of the segregate is comparable to GRID topology, in some instances. We also demonstrate that the effect of single channel interference has been minimised and the throughput of the network has been increased, far more when multiple channel allocations are deployed within the segregate. Segregate networks are more robust in harsh environments and provide better data reliability.
\end{abstract}

\section{Introduction}

Ad-hoc wireless networks provide a means of networking together groups of computing devices without the need for any existing infrastructure. Devices automatically form a network when within range of each other, and also act as routing nodes by forwarding any packets not intended for them. This permits nodes to communicate further than their transmit power permits, and also allows and provides a more optimal use of the radio spectrum.

Common problems with wireless networks are interference, multipath and attenuation. All these prevent the wireless networks from performing to their maximum capabilities.

In this paper we examine the impact of utilising multi-channel technology. Our target is to investigate the performance of the existing GRID channel assignment protocol compared to segregated multichannel mesh network and a simple, single channel wireless network - WLAN. The term segregated means that the network is divided into smaller areas/domain and each one operates only one radio. Each node is assigned one radio frequency but each segregate part has been assigned a different radio from the others. One of the advantages of this approach is that the effect of single channel interference has been minimised as each segregate network consisted of the least number of static nodes possible spreading randomly within the tested area. Apart from that, we were able to duplicate the data and send the same data through different segregate areas simultaneously, to overcome the interference in harsh environments.

\section{Literature Review}

There are many proposed solutions for the MAC and the network layers, new routing algorithms as well as existing algorithms improved ones.

Node placement and deployment play a crucial role to the network stability and performance. When nodes are placed in a proper way taking into consideration other environment characteristics such as sources of interference and area morphology, it is easier to adjust the deployed wireless network to those needs to achieve maximum operability and performance.

To reduce interference, neighboring nodes should operate in different frequency channels. For example the IEEE $802.11 \mathrm{~b}$ standard for wireless LANs can 
operate simultaneously in three non-overlapping channels (1, 6 and 11) [1] without each node interfering with each other. There are two kinds of infrastructures of wireless LANs, the single-hop and the multi-hop. Each client inside the single-hop network should be within the range of the access point. The limitations that appear are can be reduced load traffic management and the need for a large number of backbone nodes to relay the traffic to the main network, which in most cases is a wired one.

On the other hand, in the multi-hop multichannel infrastructure, a node may find many routes to different access points, potentially operating on different channels. Thus each node must select the most appropriate route in order to achieve the best possible performance.

One approach is to use a single Network Interface Card (NIC) and appropriately manage the channels in use. The NIC should switch from one channel to another every time the node initiates a communication by choosing a channel $k$ from a pool of available channels, and hence avoiding interferences. Kyasamur and Vaidya, [2] proposed a routing and channel assignment protocol, which is based on traffic load information. The proposed protocol successfully adapted to changing traffic conditions and improved performance over a single-channel protocol adopting random channel assignments. Bahl [3] suggested a linklayer protocol called SSCH that increases the capacity of an IEEE 802.11 network by utilizing frequency diversity. Another approach of the multi-channel subject was to install multiple NICs with each one operating with different channels. Based on that, it suggested [4] the development of a wireless mesh network architecture called Hyacinth.

In the next chapter there is a presentation of the network architectures used during our simulations, their characteristics and the differences between them.

\section{Systems Architecture}

In the case of harsh industrial environments, the problems encountered can be more persistent and result in low performance. The problem of broken links has been mainly encountered by the deployment of multichannel networks. All the solutions proposed until now are really complex and it is difficult to be implemented in real life for commercial use. The proposed approach in this paper tries to provide a simpler solution which with minor and simple changes on the routing algorithm will provide an alternative to current technologies that can be easier applied. In the proposed network configuration nodes are usually fixed, in a mesh configuration and they are used to send, receive or relay information from other nodes. Information traveling across them is data captured from machinery sensors. This means that the wireless nodes perform a difficult and important task. The main problem in mesh networks is the interference between the nodes that operate on the same channel. The nodes tend to fail to transmit as their neighbors operate on the same frequency channel. The multi-channel approach solved partly this problem. However, the ability of the wireless nodes to manage efficiently their frequency channel decisions remains an issue. This paper addresses some of these issues.

GRID proposed by Tseng et al, [5] is a locationaware routing and channel protocol that enables each node to be aware of its position, through a GPS device. In our case we concentrate on the fixed nodes, architectures as out environment require restricted mobility. Our proposed approach is to divide the whole network into subnetworks, not physically, but according to the number of channels. Each subnetwork uses only one particular channel, irrelevant with the rest of the network in total.

Within a multi-channel environment the two main issues that should be addressed are: channel assignment and medium access. GRID uses the location information of the nodes to solve the channel allocation problem. The medium access can be a frequency band, either FDMA or CDMA. Disregarding technology dependence, the channel access capability can done either by using a single transceiver, the node can access only one channel at a time, either in simplex or duplex mode or by a multiple transceiver.

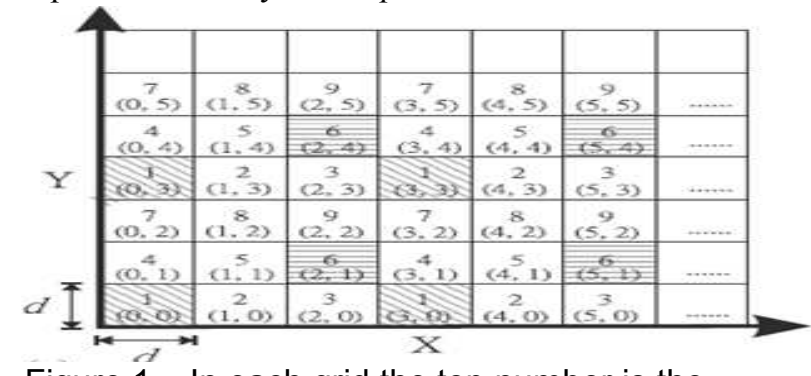

Figure 1. In each grid the top number is the channel number and the ones in the bottom are the grid co-ordinates.

The GRID is a multi-channel MAC protocol [6] able to access multiple nodes increasing the available bandwidth within the wireless network and also reducing the possibilities of contention/collision. The idea of GRID is to divide the physical area of the 
wireless network in smaller squares called grids, as in GSM. Each grid is assigned a default frequency channel for the nodes to operate in. Every node that is within this grid uses this single channel. The nodes are equipped with a GPS device and are constantly aware of their positions and select their channels according to the principles of the grid. There might be more than one node within each grid. The reason for selecting GRID is to prove that simpler routing algorithms with network segregation can achieve better results than complex and expensive proposals.

We propose a new wireless network configuration, which aims to increase the throughput of the network, overcomes some of the already mentioned problems and finally operates within acceptable delay requirements. The configuration is called Segregate wireless networks and has an analogous approach as the GRID. The nodes are fixed and spread across a bounded geographical area. The network is divided, segregated, into smaller subnetworks where each one operates with a different frequency channels. These nodes might operate in a single channel or they can be multichannel enabled, operating in channels different than the rest of the segregates. There is no communication between them as the target of the network is to transfer data from one side of the network to the other. There are usually two edge nodes that are responsible for the data transfer. These nodes are multichannel enabled and can switch from one channel to the other in order to achieve communication with all the segregates so data can travel from one side to the other following many possible routes at the same time.

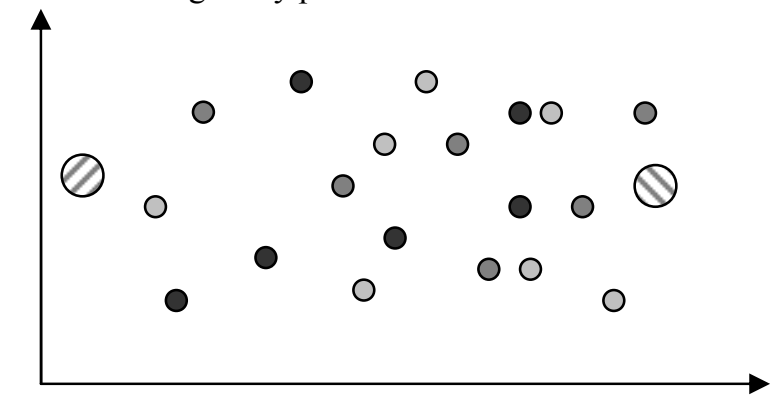

Figure 2. A segregate network of 21 nodes. The side nodes operate in all the three channels available.

After the general description of the different architectures the next section considers the detailed description of the testing and simulation.

\section{Architectures evaluation}

Both networks have been designed carefully to meet the requirements and the specifications in order to achieve the best possible approach and gather the most accurate results from the simulations. The idea behind this paper is to test both technologies in a physical environment where it would be indoors, such as an industrial building. Purpose is to gather information from one particular part of the network and transfer it on the gateway of the wireless network, where the data will be transferred to the main storage facility disregarding the wired part.

\subsection{GRID Network}

The area simulated $a$, has dimensions of $200 \times 200$ meters with a variable number of nodes $n$. Since GRID is able to divide the whole into many smaller grids, each grid should have a different channel allocated to its area. Thus we decided not to divide the area into many small grids. This way we keep the number of channels low and at the same time testing becomes easier. The number of nodes $n$ simulated range between $20 \leq n \leq 105$.

On the other hand the grid size was given two different values. Testing has been implemented for grid size of $g=10$ and $g=40$ meters, keeping the same total area dimension $a$ and frequency channels $k$. The $g$ defines the length of each side of every grid and it is calculated into meters. Four frequency channels were deployed inside the networks. In GRID technology the number of grids and the number of channels used are relevant and depended on each other. An example of the simulated GRID network can be seen in figure 3 . In both networks we kept the same density $b$, which is given from the following equation

$$
\mathrm{b}=\mathrm{n} / \mathrm{a}
$$

Further more, the transmission power $P t$ is related to the range of transmission $d$ of the nodes and is expressed as:

$$
d=\frac{\sqrt{\frac{P_{t}}{P_{r}} c^{2}}}{4 \pi f}
$$

In the above equation $\mathrm{Pt}$ is the power of the transmission, $\operatorname{Pr}$ is the power of reception and $\mathrm{f}$ is the frequency. During the simulations, all the nodes were placed randomly inside the area a. By increasing $n$, more grids were occupied by at least one or more nodes. As a result, each node could transmit with less interference. 


\subsection{Segregate Network with single channel operating subnetwork}

In this scenario we deploy always the corresponding number of nodes as in the GRID one and keeping the same physical characteristics of the simulated area. The difference from the GRID is that the nodes are divided into groups depending on the number of nodes and the number channels used. Since $\mathrm{k}$ is constant in the scenarios the only thing that changes is the number of nodes within each segregate subnetwork. Each subnetwork operates in a single channel, which is different than the rest, for example one subnetwork operates on channel $k l$ the next one operates on channel $k 2$ and so on. This ensures that there is no communication between the segregated parts of the whole network. The only different network characteristic from GRID is the way that channels are allocated to the nodes. Inside the segregate network channels are assigned according to the total number of nodes and channels used. It is a random technique, which just ensures that each subnetwork has the same number of nodes as the rest. This is done mainly to balance the traffic in each segregate network. There are cases where the number of the nodes for each segregated differs. This causes spreading around the physical area and their range would be smaller than the distance between them.

\subsection{Segregate Network with multiple channel operating subnetworks}

In this case, each subnetwork is operating into more than one frequency channel. Again the frequencies in one subnetwork $\{k 1, k 3, k 5 \ldots k n\}$ differ from the frequencies operating in the other $\{k 2, k 4, k 6 \ldots k n+1\}$. Again, the number of channels existing in one subnetwork will be the same to all the rest.

According to the scenario, a modification was made in the way the nodes switch channels during data transmission. The switching technique is based on modulo algorithm, and it is explained in more details in [7].

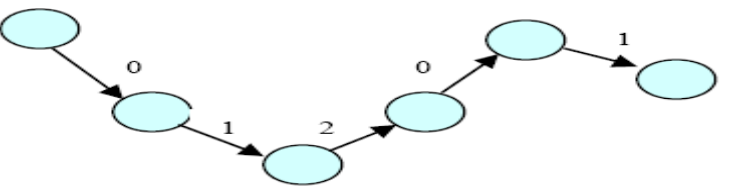

Figure 3. Modulo channel allocation algorithm.

A node, upon receiving data packet on a channel $\mathrm{k}$, transmits it on the next channel $k+1$, where $k+1$ is next channel greater than the current one in rank. In general, the channel that is in use at hop $h$, given a starting channel $k$ and $e$ channels available can be expressed as

$$
f \mathrm{~h}+1=(\mathrm{h}+\mathrm{k}) \bmod \mathrm{e}
$$

When a transmission is initiated, a random channel $\mathrm{k}$ is selected to lower the possibilities of other nodes selecting the same channel. As the selection is random there is no guarantee that the same channel will not be chosen. So more the channels used less the chances to reuse the same one.

Modulo performs better when the nodes are placed in a chain topology. On any other topology its performance decreases as it experiences interference from intersecting and adjacent traffic flows. Until now modulo had only been tested in chain topology in its simplest form [7]. However, in the case of segregated networks these problems were eliminated. We managed to overcome the interference from intersecting traffic flows as each segregate network is operating on different frequency channels. Since AODV, Ad hoc On-Demand Distance Vector, sets up a route until the transmission is finished, only one segregate network will be used to transfer the data. If another node tries to set up a transmission at the same time, AODV will establish a different route from the one already established, using a different subnetwork and since each subnetwork operates on different frequency channels, intersecting interference is reduced.

The route set up is decided by the routing protocol used and in our case it is AODV [8] multichannel enabled [9]. Modulo is only responsible for the allocation of the channels between the nodes during the transmission. More than one node of each subnetwork is able to listen to the side nodes, reducing the chances for a broken link between them. Every time a side node sends data, it selects the channel randomly without satisfying any criteria as long as the other nodes are not busy. A graphical representation of a segregate network used can be seen in figure 4 .

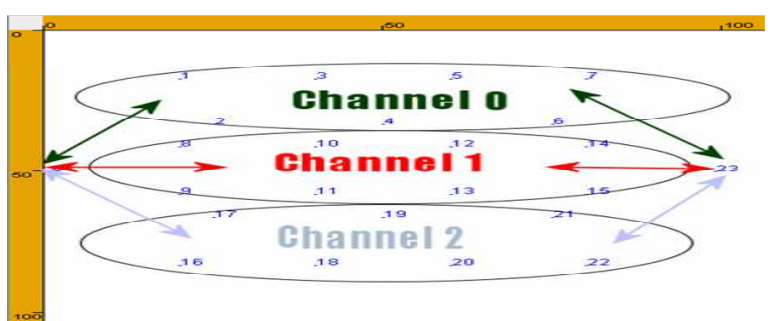

Figure 4. A segregated network, using three channels. 
When the left side node starts a transmission, AODV will set up a route through one the available subnetworks and in the case of figure 4 it has three options. If there was one more node that would be able to listen to all the available channels and requested a transmission AODV would set up a route using one of the other subnetworks. This way multiple simultaneous transmissions would be achieved and they would not affect each other. On the other hand if only one channel was present and was used by all the nodes, the effect of interference would slow down the network by reducing the throughput and by increasing the delay. Simultaneous transmissions would be out of question as it would require more than one channel. Also it should be pointed out that with the help of modulo interference within a subnetwork would be also minimized because of the frequency hopping that takes place during the transmission.

The advantages of this approach are the increase of the total bandwidth available in the network but also reduction of data losses, and thus achieving a greater reliability. Each segregate network provides a different route utilizing the maximum bandwidth. Another issue to taken into consideration is the transmission power of the nodes Pt. In order for the network to perform at its maximum, the transmission power is adjusted accordingly, $-2 \mathrm{~dB} \leq \mathrm{Pt} \leq-6 \mathrm{~dB}$. For example when $\mathrm{n}=$ 25 , the transmission power of $-4 \mathrm{~dB}$ that minimizes the delay.

Any mobile nodes such as laptops are able to move easier within and achieve better connections with the fixed nodes/access points.

\section{Results}

The simulator used for this purpose was GlomoSim [10], a parallel discrete event similar that uses Parsec. The following graphs present the results acquired from the comparison of the three different networks. The area is a $200 \times 200$ meters, using $802.11 \mathrm{~b}$ and maximum bandwidth is $11 \mathrm{Mbps}$. There are the two side nodes at the two opposite sites of the physical area. In order to increase the traffic and the load of the network, we designated the two side nodes to send data to each other at the same time. The duration of the simulation is set to 15 minutes. The application to generate the traffic is a constant bit ratio generator that sends data during all the simulation time. The parameters of the network that are compared are the average delay, the average throughput and the delivery ratio of the data within the network.

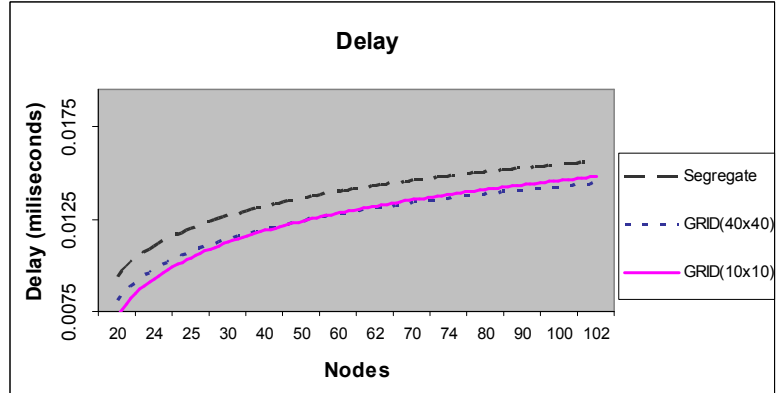

Figure 5. Average delay of the networks for a variable number of nodes.

As we can see from figure 5, the average delay of the segregate network is slightly higher than the GRID technology. This is mainly due to the lack of an appropriate routing protocol. Although AODV does perform pretty well within a single-receiver node network, in a multichannel segregate network its performance is not the best possible. Since the data has to go through many routes, in case of congestion in one route there is no way for the route to be relayed to another subnetwork where the load is quite lower. In this case the AODV is not very suitable for the current segregate approach. The network might function better if there was a more appropriate routing technique. Nevertheless even at the current form the results are quite promising regarding the delay parameter.

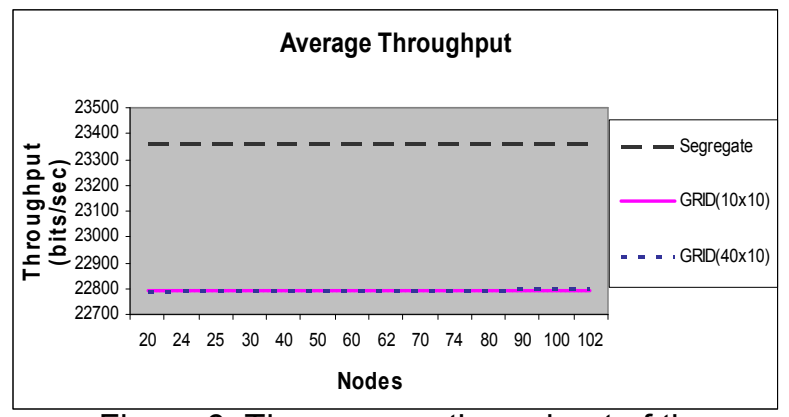

Figure 6. The average throughput of the networks for variable number of nodes.

One of the main advantages and capabilities is the increase of the throughput within the network. According to our requirements, the segregate network provides an increased throughput for the same sending/receiving configuration. This happens mainly because the multiple routes provided by the subnetworks and that the sending nodes can switch within the different frequency channels with minimum delay. It should be noticed that we were not able to define a maximum load overflow, which would result in routing data through another path the average throughput of the network remains quite high. If we 
were able to set a load overflow and start routing data through less congested subnetworks, then we could see a slight increase to the throughput having a better utilization of the different subnetworks and possibly a delay decrease.

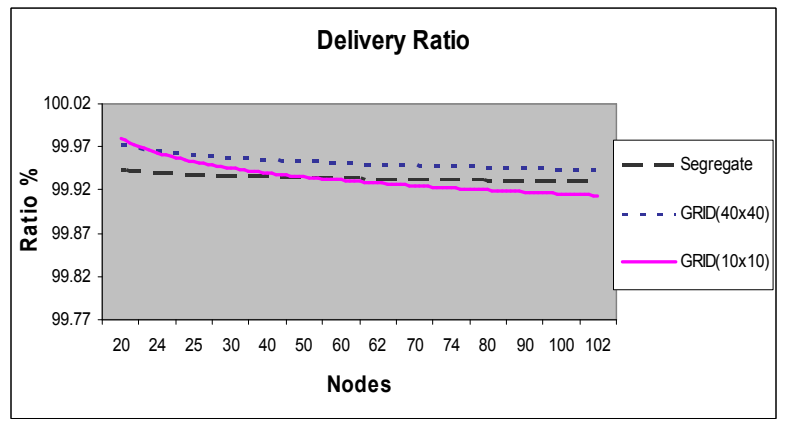

Figure 7 . The delivery ratio of the networks

In figure 7 shows the QoS of the segregate and the GRID networks. The delivery ratio gives us the sent/received ratio. The ratio of segregate network is quite constant and does not have big deviation as the nodes increase. Although it might not reach the $100 \%$ it is very close to it and generally it performs better than the GRID protocol, which shows greater deviations even if it reaches the $100 \%$ reliability.

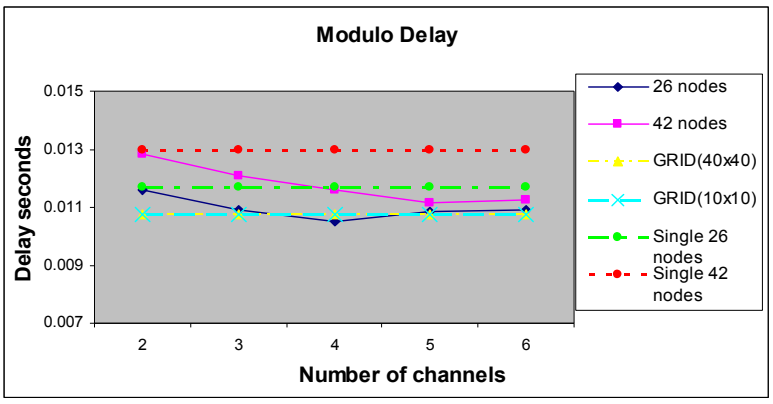

Figure 8. Average delay inside the subnetworks using modulo.

In figure 8 we have divided the network into four subnetworks. In each subnetwork we used an increasing number of channels, starting with 2 channels to a maximum of 6 . As can it can be seen from the graph, the delay tends to decrease as the number of channels increases, until 4 channels are deployed for a 26 nodes network and 5 channels for 42 nodes. The increase of the number of channel above 6 does not bring any improvement to the delay. The results compared to the single channel segregate network are much better which was something expected, as the interference within the subnetwork is reduced.

Segregate networks are an idea trying to exploit the spatial re-use advantages incorporating the maximization of the network capacity by the increase of the available channels. Combining those two methods into a single network could possibly achieve great results regarding network performance and stability. After all, the testing is done under certain network requirements and thus certain solutions that would perform better in different scenarios, but in this case they might not return the best possible results. All these tests are performed in order to identify the advantages and the disadvantages of ideas proposed currently by researchers worldwide.

\section{Conclusion and future work}

In this paper we evaluated two different network architectures, the Segregate and the GRID. Both are multi-channel enabled technologies used to transfer data within an industrial environment. We are proposing the segregate configuration and we test it against GRID proposed technology. From the results we can see that the general performance of our proposal is very satisfactory as it provides a higher throughput within the network and a better stability to the variations of the sending/receiving ratio. This is achieved by taking advantage of all the benefits of mesh networks and network segregation. In another work it was proved [11] that the number of collisions inside the network is significantly reduced and comes in accordance with the results presented previously.

This paper is a sequel of our previous publication [12], where we compared the segregate network with a uniform multi-channel network. The idea of the segregated is in its initial stages and there are thoughts and undergoing implementations to improve it by setting a better routing algorithm more suitable for this kind of network configuration. Also other existing routing protocols will be tested apart from the AODV.

\section{References}

[1] IEEE Standard for Wireless LAN-Medium Access Control and Physical Layer Specification, P802.11, 1999

[2] P. Kyasamur, N. H. Vaidya, Technical Report, October 2004.

[3] P. Bahl, R. Chandra, J. Dunagan, "SSCH: Slotted seeded Channel Hopping for Capacity Improvement in IEEE 802.11 Ad-Hoc Wireless Networks", ACM Mobicom, 2004.

[4] A. Raniwala, T. Chiueh, "Architecture and Algorithms for an IEEE 802.11-Based Multi-Channel Wireless Mesh Network", IEEE Infocom, 2005. 
[5] Y. Tseng, C. Chao, S. Wu, J. Sheu, "Dynamic Channel Allocation With Channel Awareness for Multi-hop Mobile Ah Hoc Networks", Computer Communications, 25(7), May 2002, pp. 676-688.

[6] Y. Tseng, C. Chao, S. Wu, J. Sheu, “An Efficient MAC Protocol for Multi-Channel Mobile Ad Hoc Networks Based on Location Information", Int'l Journal Communication Systems, Vol. 19, 2006, pp. 877-896.

[7] M. Adda, G. H. Owen, M. Kassasbeh, A. Paraskelidis, and A. Peart, "Communication issues in Large Scale Wireless Ad hoc Networks," in International Conference on Computer Science and Information Systems, Athens, Greece, July 2005.

[8] C. Perkins, E. Belding-Royer, S. Das, "Ad hoc OnDemand Distance Vector (AODV) Routing", in Ietf RFC 3561, July 2003.

[9] Owen, G., Adda, M., "Modulo and Grid Based Channel Selection in Ad Hoc Networks", in IADAT-ten, September 2006.

[10] UCLA Parallel Computing Laboratory, "Global Mobile Information Systems Simulation Library", Available: http://pcl.cs.ucla.edu/projects/glomosim

[11] A. Paraskelidis, M. Adda, "The Performance of Segregate WLAN inside a Noisy Industrial Environment" in International Conference on Computer Science and Information Systems, ATINER. Athens Greece, July 2008.

[12] A. Paraskelidis, M. Adda, "An evaluation of uniform multi-channel network versus multi-channel segregate network", in IADAT-ten2006, September 2006. 